\title{
ORGANIZACIONES INTELIGENTES Y SU INCIPIENTE INCURSIÓN EN LA ESFERA TURÍSTICA. UNA APROXIMACIÓN AL ESTADO DEL CONOCIMIENTO
}

\author{
Gema Simona Navarrete de la O* \\ https://orcid.org/0000-0002-1909-0814 \\ Arlén Sánchez Valdés** \\ https://orcid.org/0000-0001-5241-4123
}

RECIBIDO: Junio 2021 / ACEPTADO: Octubre 2021 / PUBLICADO: Enero 2022

Como citar: Navarrete, Gema; Sánchez Valdés, Arlén. (2022). Organizaciones inteligentes y su incipiente incursión en la esfera turística. Una aproximación al estado del conocimiento. Telos: revista de Estudios Interdisciplinarios en Ciencias Sociales, 24 (1), Venezuela. (Pp. 100-122).

DOI: www.doi.org/10.36390/telos241.07

\section{RESUMEN}

En los últimos años autores como Aksu y Özdemir (2005); Bayraktaroglu y Ozen Kutanis (2003); Hallin y Marnburg (2008); Kraleva (2011); Ortega Herrera y Sánchez Ramos (2018); Valecillos y Quintero (2007); han llegado a la conclusión de que las empresas se han visto en la necesidad de ajustarse a los constantes cambios que demandan los entornos políticos, económicos y sociales, obligándolas a invertir en sus recursos internos como el capital intelectual, talento humano, conocimientos y tecnología. Por lo cual, a través del análisis de 50 artículos entre los años de 1996-2020, clasificados en perfiles temáticos: organizaciones tradicionales, organizaciones que aprenden u organizaciones inteligentes, análisis organizacional de las organizaciones inteligentes, así como organizaciones turísticas inteligentes, obtenidos de bases de datos como ScienceDirect, Dianelt y Scielo; motores de búsqueda como Mendeley y Google Scholar; y finalmente con editoriales mundiales como Emerald Insight, además de utilizar el software VOSviewer para obtener datos más precisos de las investigaciones, se logró identificar que para eliminar la incertidumbre y formar empresas competitivas, éstas se deben transformar en organizaciones inteligentes que generen un equilibrio entre la oferta y demanda, con el objetivo de ganar clientes satisfechos. Siendo que, esta transformación empresarial se logra a través del aprendizaje, el fortalecimiento de capacidades de innovación, el intercambio de conocimiento a través de un entorno colaborativo, y un compromiso de los empleados en todo el proceso de producción, las áreas funcionales, los liderazgos empresariales y las políticas organizacionales, que en conjunto forman una empresa inteligente, que aplicada a una empresa

\footnotetext{
*Egresada de la Licenciatura en Turismo, Universidad Autónoma del Estado de México. Correo electrónico: gemanava96@gmail.com ** Licenciatura y maestría en Administración. Doctorado en Estudios Turísticos. Docente en la Universidad Autónoma del Estado de México. Correo electrónico: asanchezva@uaemex.mx
} 
turística tendrá como resultado la calidad de los productos o servicios satisfaciendo las necesidades de los clientes, incluso rebasando sus expectativas, logrando así ventajas competitivas dentro de un mercado global más complejo.

Palabras clave: aprendizaje, adaptabilidad, ventajas competitivas, organizaciones tradicionales, organizaciones turísticas inteligentes.

\title{
Intelligent organizations and their incipient foray into the tourism sphere. An approach to the state of knowledge
}

\begin{abstract}
In recent years, authors such as Aksu and Özdemir (2005); Bayraktaroglu and Ozen Kutanis (2003); Hallin and Marnburg (2008); Kraleva (2011); Ortega Herrera and Sánchez Ramos (2018); Valecillos and Quintero (2007); have come to the conclusion that companies have found it necessary to adjust to the constant changes demanded by the political, economic and social environments, forcing them to invest in their internal resources such as intellectual capital, human talent, knowledge and technology. Therefore, through the analysis of 50 articles between the years 1996-2020, classified in thematic profiles: traditional organisations, learning organisations or smart organisations, organisational analysis of smart organisations, as well as smart tourism organisations, obtained from databases such as ScienceDirect, Dianelt and Scielo; search engines such as Mendeley and Google Scholar; and finally with global publishers such as Emerald Insight, in addition to using VOSviewer software to obtain more accurate research data, it was possible to identify that to eliminate uncertainty and form competitive companies, these must be transformed into smart organisations that generate a balance between supply and demand, with the aim of gaining satisfied customers. This business transformation is achieved through learning, the strengthening of innovation capabilities, the exchange of knowledge through a collaborative environment, and a commitment of employees throughout the production process, functional areas, business leadership and organisational policies, which together form an intelligent company, which applied to a tourism company will result in the quality of products or services satisfying the needs of customers, even exceeding their expectations, thus achieving competitive advantages in a more complex global market.
\end{abstract}

Keywords: learning, adaptability, competitive advantages, traditional organizations, intelligent tourism organizations.

\section{Introducción}

En los últimos años, el sector turístico se ha visto impactado por un sin número de fenómenos que han afectado su competitividad, tales como: el surgimiento de plataformas de economía colaborativa, cambios sociales, ambientales, y más recientemente la crisis mundial derivada del gran confinamiento provocado por el COVID-19 (por sus siglas en inglés, Coronavirus Disease 2019). En respuesta, han surgido varios intentos para estudiar el aprendizaje y la inteligencia en las empresas turísticas; aunque todavía la investigación es incipiente y escasa. No obstante, se logra rescatar lo mencionado por Chacón León (2017), las organizaciones inteligentes se adaptan mejor a los cambios logrando en su mayoría impactar 
Organizaciones inteligentes y su incipiente incursión en la esfera turística. Una aproximación al estado del conocimiento

fuera y dentro de la empresa, que por el contrario, las organizaciones tradicionales son susceptibles a no tener posibilidades de permanecer en el mercado, debido a la incertidumbre de adaptarse a las nuevas tendencias, ya que se centran en procesos cerrados y jerarquizados, los cuales, se han venido manejando desde años por creencias, ideas o perjuicios de la organización, así como la falta de desarrollo en la cultura organizacional que refleje la profesionalidad de la empresa como de su personal, quienes den una respuesta oportuna ante su competencia y la demanda de los productos o servicios. Por tal razón, solo aquellas que se autoanalicen, comprendan los cambios de su entorno, aprendan de sí mismas, desarrollen nuevas habilidades y competencias, así como una mejor estructura organizacional, tendrán ventajas competitivas a largo plazo.

Tradicionalmente, las organizaciones inteligentes también llamadas organizaciones que aprenden $u$ organizaciones autocualificantes, son aquellas empresas que están involucradas en un proceso de cambio y transformación mediante el aprendizaje. La diferencia entre una organización tradicional de una organización inteligente es la forma en cómo crean modelos para afrontar los cambios y evoluciones internas que son un reflejo de las necesidades externas, al mismo tiempo que, la organización de aprendizaje es un esfuerzo para continuamente aprender, mejorar, gestionar, transmitir y transformar el rendimiento individual y organizacional a través del aprendizaje y así acelerar el enfoque de crecimiento a raíz del conocimiento para desarrollar experiencias que le permiten adaptarse proactivamente a un entorno cambiante (Solf Zárate, 2007; Weldy, 2009; Chávez Hernández y Torres Sanabria, 2012; Gotcheva, Watts y Oedewald, 2013).

Aunado a ello, las organizaciones turísticas inteligentes no se gestionan por el tipo y la cantidad de recursos tecnológicos, infraestructura, humanos 0 intelectuales con los que cuenta, sino en la capacidad de la empresa para gestionarlos y con ello crear ventajas competitivas que proporcionen algo diferente a sus consumidores, que muestren calidad en los productos y servicios con un valor superior a la de sus competidores, que dependerá de la capacidad de los empleados para satisfacer las necesidades de los clientes y la aptitud de intercambiar tanto la información como los conocimientos para crear ideas innovadoras que mejorarán el desempeño organizacional en su conjunto (Bayraktaroglu y Ozen Kutanis, 2003; Aksu y Özdemir, 2005; Hallin y Marnburg, 2008; Kraleva, 2011; Popescu, Chivu, CiocârlanChitucea, Popescu y Georgel, 2011; Mahapa, 2013; Suveatwatanakul, 2013; Ortega Herrera y Sánchez Ramos , 2018; González Macías, Guerrero Rodríguez y Vidaurri Aréchiga, 2019). De modo que, siendo un tema extenso, multidisciplinario y difícil de comprender, resulta fundamental dilucidar cómo las empresas aprenden y generan valor a través de la restructuración de sus procesos internos y externos para finalmente entender los desafíos para llegar a la competitividad, los cuales permitirán transformar a las empresas turísticas en inteligentes.

Por tal razón, el presente trabajo realiza una revisión de literatura con el propósito de determinar el estado actual del conocimiento en cuanto a las organizaciones inteligentes y su enfoque en las empresas turísticas ya sean hoteles, restaurantes, agencias de viajes como aerolíneas. Por tanto, el artículo se estructura en cinco apartados, el primero busca construir una definición propia de las organizaciones inteligentes a partir de la transformación empresarial de las últimas décadas. Posteriormente, se incluye el apartado de las organizaciones tradicionales 
vs las organizaciones inteligentes desde la cultura organizacional a las áreas funcionales, para identificar claramente las diferencias entre cada una de ellas y hacia dónde van dirigidas de acuerdo con sus visiones, estrategias, incluso objetivos organizacionales y consecutivamente analizar la incipiente incorporación del turismo al entorno de las organizaciones inteligentes. Por consiguiente, se encuentra la metodología del trabajo, las conclusiones y futuras líneas de investigación.

\section{De organizaciones tradicionales a inteligentes. La construcción de una definición propia a partir de la transformación empresarial de las últimas décadas}

Para entender el origen del concepto de las Organizaciones Inteligentes (OI) Valecillos y Quintero (2007), identificaron los paradigmas administrativos de cuatro décadas que han marcado a las organizaciones, que compone desde los años 50 del siglo pasado, donde la ideología dominante era que cualquier problema de la empresa se podía resolver modificando el organigrama, sin embargo, se comprobó como a pesar de estar bien organizadas, fracasaban; así, en la década de los 60 y 70, se apostó y se evidencio que cualquier estrategia desarrollada por la empresa debía ser llevada a cabo por personas comprometidas, quienes aporten conocimiento y dedicación al trabajo mediante el liderazgo, lo cual, si lograba desarrollar estas estrategias dentro de la organización y eran superiores a la de sus competidores tendrían éxito, las demás fracasarían. Esto llevo a que para los años 80 surgiera un nuevo paradigma; el de la cultura organizacional. Según éste, para lograr el éxito de la empresa no solo necesitaba una buena organización y estrategia, sino un equipo de gente capaz de imaginar, formular e implementar la estrategia, incorporando al recurso humano como actor clave en todo el proceso.

Como resultado, las teorías de estas dos últimas décadas dan origen al enfoque europeo desarrollado por John Burgoyne, Mike Pedler y Tom Boydell, quienes mencionan que las empresas que se vuelvan inteligentes son capaces de adaptarse a las exigencias cambiantes del mercado y de los sistemas sociales y políticos, así como el conocimiento transformado en aprendizaje (Talla Ramos, 1998). Mientras tanto, en la década de los noventa, la calidad total dio respuesta a la optimización del proceso de producción y el humano, los cuales se centran en satisfacer las necesidades de los clientes a través de un equilibrio entre oferta y demanda, siendo esto en conjunto con la publicación de Senge (1990) "La quinta disciplina", lo que perfila el enfoque estadounidense (Valecillos y Quintero, 2007). En ambas vertientes se ve al aprendizaje individual y organizacional como un elemento esencial para convertirse en una organización inteligente o de aprendizaje, facilitadora del flujo de conocimientos, siendo este la base para que la empresa esté preparada a adaptarse a los cambios internos y externos, permitiendo alcanzar una transformación continua, así como ventajas competitivas.

En realidad, más allá de las teorías prevalecientes en los hemisferios del mundo, es a comienzos de este siglo que empezó a madurar el paradigma de la "organización inteligente" o la "organización que aprende" (Valecillos y Quintero, 2007), como resultado de los procesos de cambio de las organizaciones exitosas de décadas pasadas, las cuales se estaban moviendo hacia un enfoque "suave" con cualidades como la perspicacia, aprendizaje, intuición y creatividad para lograr una ventaja competitiva y, en última instancia, la supervivencia (Gardiner y Whiting, 1997).

Dos décadas pasaron desde que se inicia con el trabajo formal para reinventar la estructura organizacional, los procesos de gestión y aprendizaje. Donde se ha podido consensar 
Organizaciones inteligentes y su incipiente incursión en la esfera turística. Una aproximación al estado del conocimiento

como una organización inteligente se basa en el aprendizaje individual con sentido de humanización, implementación de información y conocimientos utilizados para renovar tanto las aptitudes como actitudes de los empleados, impulsando a fortalecer y mejorar el entorno organizacional volviéndolo sólido, adaptable y duradero, ya que esto le permitirá a la empresa avanzar rápidamente en el mercado, teniendo resistencia ante los cambios competitivos a nivel global, los cuales son un reflejo de la capacidad de agregarle valor a los productos o servicios que ofrece.

Más aún, las organizaciones inteligentes se vuelven competentes al descubrir, crear, adquirir, transferir, modificar, evaluar y reforzar sus conocimientos valiosos y acciones que sean necesarias para transformar información en aprendizaje, llevando a la empresa a comprender ¿qué es lo que hace?, ¿cómo lo hace?, ¿con que propósito? y ¿por qué?, visualizando y haciendo un contraste de sus situaciones pasadas con su entorno real, para generar estrategias en la búsqueda de la competitividad y así afrontar los retos diarios (Hill, 1996; Talla Ramos, 1998; Pérez-Soltero, 2006; López-Zapata, García-Muiña y García-Moreno, 2016).

\section{Organizaciones tradicionales vs organizaciones inteligentes, de la cultura organizacional a las áreas funcionales}

La diferencia fundamental entre una organización tradicional y una inteligente es la forma en cómo crean modelos mentales, los cuales son suposiciones o perjuicios arraigados con el paso del tiempo en la mente de la sociedad como de las empresas. Por tanto, las empresas inteligentes los utilizan de tal forma que funja como una herramienta para resolver problemas de la realidad permitiendo encontrar soluciones y ayude a la toma de decisiones racionales, empezando a cuestionarse todo lo que pasa alrededor de la organización, haciendo eficaz la comunicación del personal con la empresa, promoviendo así el aprendizaje para afrontar sus transformación, que darán como resultado la calidad de productos y servicios (Aksu y Özdemir, 2005; Bahar Aşcı, Zehra Tan y Altıntaş, 2016).

Los tres elementos medulares que definen la orientación de una empresa son: la visión, la cual se apoya del líder de la empresa quien promueve el compromiso de su personal y el intercambio de conocimientos, para lograr los objetivos corporativos que llevan a un fin específico; dirección, siendo este el camino y los procesos que se deben de implementar o mejorar para alcanzar las metas en un determinado plazo, y de esta manera darle un seguimiento continuo para evaluar su nivel de efectividad; finalmente su personal, que es el capital de talento humano el cual promueven el conocimiento de la empresa, llevan a cabo estrategias para la mejor competitividad en el mercado y quienes hacen más tangible los resultados debido a la toma de decisiones constantes para la resolución de problemas.

De hecho, tal como se observa en la tabla 1, en las organizaciones tradicionales la alta dirección define la visión y toma decisiones como la encargada de resolver los conflictos, mientras que, en las de corte inteligente, se apuesta por el conocimiento y la responsabilidad grupal mediante el aprendizaje.

Tabla 1. Organización tradicional vs organización inteligente \begin{tabular}{l|l} 
Organización tradicional & Organización inteligente
\end{tabular} 


\begin{tabular}{|c|c|c|}
\hline $\begin{array}{l}\text { Orientación } \\
\text { general }\end{array}$ & $\begin{array}{l}\text { La visión es definida por la alta } \\
\text { gerencia. }\end{array}$ & $\begin{array}{l}\text { Hay una visión compartida, pero la alta } \\
\text { gerencia es responsable de desarrollar } \\
\text { esta visión. }\end{array}$ \\
\hline $\begin{array}{l}\text { Diseñando y } \\
\text { practicando } \\
\text { las decisiones }\end{array}$ & $\begin{array}{l}\text { La alta dirección decide qué hacer. } \\
\text { El resto de la organización actúa } \\
\text { de acuerdo con las decisiones de } \\
\text { la alta dirección. }\end{array}$ & $\begin{array}{l}\text { La toma de decisiones y la práctica se } \\
\text { pueden ver en absolutos niveles de la } \\
\text { organización. }\end{array}$ \\
\hline $\begin{array}{l}\text { La naturaleza } \\
\text { del } \\
\text { pensamiento } \\
\text { organizativo }\end{array}$ & $\begin{array}{l}\text { Cada persona es responsable de } \\
\text { sí misma; generalmente se centra } \\
\text { en el desarrollo de las habilidades } \\
\text { individuales. }\end{array}$ & $\begin{array}{l}\text { Cada } \\
\text { planifi }\end{array}$ \\
\hline $\begin{array}{l}\text { Solución de } \\
\text { conflictos }\end{array}$ & 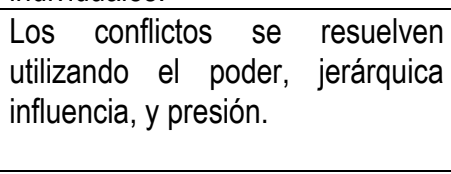 & $\begin{array}{l}\text { Los conflictos se resuelven mediante la } \\
\text { integración de diferentes perspectivas } \\
\text { del personal a diferentes niveles, } \\
\text { coordinación y trabajo en equipo. }\end{array}$ \\
\hline $\begin{array}{l}\text { Liderazgo y } \\
\text { motivación }\end{array}$ & $\begin{array}{l}\text { El papel del líder es definir la } \\
\text { visión, recompensa y sistema de } \\
\text { castigo, así como el control } \\
\text { detallado de actividades de } \\
\text { personal. }\end{array}$ & $\begin{array}{l}\text { El papel del líder es formar la visión y } \\
\text { el empoderamiento al utilizar el } \\
\text { liderazgo carismático, ya que el líder } \\
\text { fomenta la toma de decisiones } \\
\text { efectivas. }\end{array}$ \\
\hline
\end{tabular}

Fuente: Aksu y Özdemir (2005).

Autores como Slater y Narver (1995); Stonehouse y Pemberton (1999); Kraleva, (2011); Suveatwatanakul, (2013), recalcan que, a diferencia de las organizaciones tradicionales, la organización inteligente desarrolla una ventaja competitiva por las siguientes razones:

- Ejerce una planificación con base a una visión.

- Crea equipos capaces de toma de decisiones y de resolver tareas.

- Comprende el mercado actual.

- Crea una arquitectura de aprendizaje, donde se adquiera nuevos conocimientos.

- Promueve la gestión del conocimiento.

- La empresa es flexible y adaptable a los nuevos desafíos.

- Añade valor a los productos y servicios ante los ojos del consumidor.

Por tal motivo la organización inteligente es capaz de transformar la información en conocimiento útil para la empresa, permitiéndole desarrollar competencias y habilidades en todos sus trabajadores, llevándola a tomar decisiones creativas que revolucione la cotidianidad en algo innovador para satisfacer a los consumidores con constantes cambios y exigencias. Por esta razón, Rodríguez Cruz (2016), menciona como las organizaciones inteligentes son capaces de adaptarse y desarrollar competencias duraderas y adaptables ya sean internas o externas, a corto, mediano y largo plazo, lo que impulsa el alto rendimiento organizacional, porque de ahí se definirán los objetivos, las estrategias, técnicas y procedimientos hacia un crecimiento empresarial, con beneficios y fortalezas organizacionales.

\section{Fundamentos para la transformación a una organización inteligente}


Organizaciones inteligentes y su incipiente incursión en la esfera turística. Una aproximación al estado del conocimiento

Diversos han sido los enfoques que se han utilizado para describir y analizar una organización inteligente 0 que aprende, la cual, desarrolla capacidades y se establece en un mundo donde la incertidumbre como los nuevos intereses sociales y tecnológicos son totalmente dinámicos (Mendoza Pineda, 2011; Brătianu, Vasilache y Jianu, 2006). Por esta razón, con el paso del tiempo diversos autores han determinado que debe existir un desarrollo organizacional contundente y eficaz dentro de la organización inteligente, la cual ayude a evolucionar interna y externamente, por ello en la tabla 2 se observan los principios fundamentales considerados desde los primeros estudios en el tema hasta la actualidad.

Tabla 2. Evolución de los principios y enfoques de una empresa Inteligente; integración del concepto a partir del análisis de las áreas funcionales, liderazgos empresariales y un entorno cambiante (1997-2016)

\begin{tabular}{|c|c|}
\hline $\begin{array}{l}\text { Gardiner y } \\
\text { Whiting (1997) }\end{array}$ & $\begin{array}{ll}\text { 1. } & \text { Aprendizaje y autodesarrollo individual } \\
\text { 2. } & \text { Estrategia de aprendizaje } \\
\text { 3. } & \text { Clima de aprendizaje } \\
\text { 4. Uso de la información } \\
\text { 5. Empoderamiento } \\
\text { 6. Vínculos con el entorno externo }\end{array}$ \\
\hline $\begin{array}{l}\text { Talla Ramos } \\
\text { (1998) }\end{array}$ & $\begin{array}{l}\text { 1. Comparten una visión } \\
\text { 2. Actúan mediante un sistema de corresponsabilidad y compromiso } \\
\text { mutuo } \\
\text { 3. Creen en su potencial conjunto } \\
\text { 4. Comparten la información y las ideas } \\
\text { 5. Avanzan rápidamente } \\
\text { 6. Se transforman }\end{array}$ \\
\hline $\begin{array}{l}\text { Matheson y } \\
\text { Matheson } \\
\text { (2001) }\end{array}$ & $\begin{array}{l}\text { 1. Logran el propósito } \\
1.1 \text { Cultura de creación de valor } \\
1.2 \text { Creación de alternativas } \\
1.3 \text { Aprendizaje mutuo } \\
\text { 2. Comprender el medio ambiente } \\
2.1 \text { abrazando la incertidumbre } \\
2.2 \text { Perspectiva estratégica de afuera hacia adentro } \\
2.3 \text { El pensamiento sistemático } \\
\text { 3. Moviliza recursos } \\
3.1 \text { Toma de decisiones } \\
3.2 \text { Alineación y potenciación } \\
3.3 \text { Flujo de información } \\
\end{array}$ \\
\hline $\begin{array}{l}\text { Thomas y Allen } \\
\text { (2006) }\end{array}$ & $\begin{array}{ll}\text { 1. } & \text { Aprendizaje } \\
\text { 2. } & \text { Estructura } \\
\text { 3. } & \text { Visión compartida } \\
\text { 4. } & \text { Gestión del conocimiento } \\
\text { 5. } & \text { Estrategia }\end{array}$ \\
\hline $\begin{array}{l}\text { Valecillos y } \\
\text { Quintero (2007) }\end{array}$ & $\begin{array}{l}\text { 1. Aprendizaje organizacional } \\
\text { 2. Conocimientos individuales } \\
\text { 3. Procesos de aprendizaje colectivo } \\
\text { 4. }\end{array}$ \\
\hline
\end{tabular}




\begin{tabular}{|l|rl|}
\hline & $\begin{array}{r}\text { 5. } \\
\text { 6. }\end{array}$ & $\begin{array}{l}\text { Capacitación } \\
\text { Aprendizaje colectivo } \\
\end{array}$ \\
& $\begin{array}{l}\text { Las disciplinas de aprendizaje: visión compartida, pensamiento sistemático, } \\
\text { excelencia personal, cambio de paradigmas y trabajo en equipo }\end{array}$ \\
\hline Chávez & 1. & Aprendizaje organizacional \\
Hernández y & 2. & Aprendizaje individual \\
Torres & 3. & Sistemas y procesos \\
Sanabria, & 4. & Cultura \\
(2012) & 5. & Memoria organizacional para superar el aprendizaje \\
& 6. & Mejora continua \\
& 7. & Creatividad e innovación \\
\hline Gil López y & 1. & Potenciación de las personas y de los equipos de trabajo \\
Ruiz, (2016) & 2. & Gestión de la información \\
& 3. & Orientación del aprendizaje \\
& 4. & Transferencia de la información \\
\hline
\end{tabular}

Fuente: Elaboración propia a partir siete investigaciones (Gardiner y Whiting, 1997; Talla Ramos, 1998; Matheson y Matheson, 2001; Thomas y Allen, 2006; Valecillos y Quintero, 2007; Chávez Hernández y Torres Sanabria, 2012; Gil López y Ruiz, 2016).

Los autores citados en la tabla 2, demuestran que una organización inteligente se basa en un comportamiento de aprendizaje dinámico entre todos los individuos de una empresa, además de una participación, responsabilidad, empatía y apertura a los cambios a los que la empresa se pueda enfrentar. Sin embargo, los principios y características de una organización inteligente deben ir acompañados de auto transformación, experimentación, proyección, transdisciplinariedad, disminución jerárquica, visión de logro, financiamiento, asertividad para crear estrategias, habilidades y un principio de aprender a innovar, la cual, ayudará a desarrollar una dinámica de conocimiento capaz de construir una estructura organizacional que esté preparada a los cambios que puedan surgir repentinamente (Becerra y Sánchez, 2011).

Además, dentro de una organización inteligente se incluyen las áreas funcionales de la empresa, como el liderazgo empresarial vinculado a la cultura organizacional, debido a que transmite a los colaboradores de la manera más clara y entendible la visión de la empresa, utiliza toda la información recopilada a través de los conocimientos de los empleados como datos tangibles, para facilitar los procesos de aprendizaje organizacional, modelar e impulsar las tecnologías de la información y los canales de comunicación entre niveles organizacionales, que permita hacer una retroalimentación con todo el personal, funja como principio de aprendizaje y refuerce sus productos y servicios (Stewart, 2001; Amy, 2008; Gallego y Gil, 2012; Martínez González, Gómez Pérez y Martínez González, 2017), eliminando a su vez las barreras que reducen la eficacia, la capacidad de adaptación, aprendizaje, conocimiento y de establecer redes de la organización que detienen a la empresa a avanzar (Khan y Haleem, 2015).

De igual forma, el sistema humano-cultural, que es el modelo para generar nuevas técnicas empresariales, desarrollado a través de equipos inteligentes caracterizados por desenvolverse en un pensamiento sistémico, creativo, crítico, holístico y con el poder de dar iniciativas que ayuden a expandir el conocimiento, crear un ambiente laboral agradable, generar compromiso, detectar fallas y necesidades, así como entrenar eficazmente a los equipos de trabajo para que generen una metodología de análisis de flujos para el diagnóstico, planificación 
Organizaciones inteligentes y su incipiente incursión en la esfera turística. Una aproximación al estado del conocimiento

y seguimiento de los cambios asociados en la implementación empresarial, de tal forma que, se considere el entorno como uno de los principales drivers en el proceso de gestión, porque se relacionan de manera crítica con el desarrollo de la organización de aprendizaje, y alienta a los empleados a utilizar las instalaciones de aprendizaje capaces de superar los cambios (Gillezeau, 1999; Su-Chao y Ming-Shing, 2007; Valecillos y Quintero, 2007; Serrano Orellana y Portalanza, 2014). Dado que, con el impacto que tenga el aprendizaje, ya sea negativo o positivo, se determinaran las actitudes laborales de los individuos (Kanten, Kanten y Gurlek, 2015).

Por último, la tecnología SMAC (Redes sociales, móvil, analítica y nube) proporciona a los clientes servicios a la medida (Adamczewski, 2016), debido a que una organización no es un modelo exclusivo de un sistema tecnológico, ni tampoco representan un sistema social enfocado únicamente al personal de la empresa, sino que es un sistema donde las relaciones sociales aunado a la tecnología aumenta la eficacia de las personas y mejora el flujo de la información, que da como resultado una experiencia personal dirigido al cliente debido a que recibe un producto o servicio con un valor agregado, novedoso y competente (Bhatt, 2001).

Bajo esta circunstancia, una organización tradicional, se enfrenta ante un futuro de incertidumbre donde la única opción para obtener ventajas competitivas es gestionar los conocimientos del capital humano por medio de un aprendizaje continuo tanto individual como organizacional, garantizando el flujo de la información, así como mejor toma de decisiones, demostrando su capacidad ante los cambios, eliminando la zona de confort, dinámicas e interacciones de aprendizaje individuales como organizacionales, para que se convierta en una empresa más competente lo que podría asegurar su posición en el futuro y aumentar su ventaja competitiva (Brătianu et al., 2006; López-Zapata et al., 2016; Moh'd, Dahou y Hacini, 2016; Angulo Rincón, 2017; Ortega Herrera y Sánchez Ramos, 2018; Rojas, 2018).

Finalmente, se habla de una organización inteligente, cuando su trasformación se basa en el aprendizaje, el fortalecimiento de sus capacidades de innovación y el intercambio de conocimiento a través de un entorno colaborativo, en un sentido de responsabilidad y un compromiso de sus empleados en todo el proceso de producción donde las áreas funcionales, el liderazgo empresarial, políticas y cultura organizacional, así como un claro entendimiento del entorno, determinará el éxito o fracaso empresarial.

\section{La incursión de las Organizaciones inteligentes en el ámbito turístico}

La organización inteligente se establece en un mundo moderno donde la incertidumbre es el ente para desarrollar nuevas capacidades cognoscitivas que impulsan el crecimiento personal y grupal. Por dicho motivo esta inseguridad no se debe ver como una amenaza, sino como una oportunidad empresarial, que implemente metodologías para el diagnóstico, planificación, seguimiento y ejecución de cambios en la gestión gerencial y empresarial, que ayude a elaborar manuales de procesos así como modelos de negocios con base a información real y a las necesidades de la organización, los cuales, permitirán disminuir obstáculos, ser operativos, proactivos en la aplicación de las estrategias, además de, agregar valor y humanización tanto a los recursos de la empresa como al logro de los objetivos, porque 
garantizaran una gestión efectiva de talento humano y toma de decisiones eficaz e innovadoras (Valecillos y Quintero, 2007; Rojas, 2018; González Macías et al., 2019).

Sin embargo, en el caso de las empresas turísticas, se ha identificado como la organización inteligente o de aprendizaje es un concepto relativamente nuevo, contemporáneo y poco estudiado, el cual define cómo debe operar una organización en el negocio en constante cambio, tendiente a hacer cambios en el comportamiento de todos los empleados para generar un compromiso organizativo donde se alcancen los propósitos y objetivos de la empresa, impactando en el desempeño general de la organización (Atak y Erturgut, 2010). Aprendiendo sobre la propia experiencia y la de los demás. Por tanto, la combinación de elementos tales como los trabajadores, empresa, proveedores y principalmente los clientes en un entorno turístico, forman el concepto de organización inteligente turística, porque busca concentrar el servicio para una mejor satisfacción del cliente. El foco está en la satisfacción y calidad. Implicando una sinergia del conocimiento y las competencias de sus individuos (Kraleva, 2011). Para ello, los elementos que integran una OTI se obtienen del talento humano, la competitividad, la tecnología y la orientación hacia la industria turística, aunque pocas son las empresas que han sabido lograr compaginar estos elementos, refiriéndose a las grandes firmas (Ortega Herrera y Sánchez Ramos, 2018). Aunque cabe señalar que la gestión del conocimiento tiene un gran impacto en estas organizaciones porque comprende cinco fases: crear, validar, presentar, distribuir y aplicar, para así reflexionar y aprender (Larrota Castro, 2012), debido a que es mejor invertir en los conocimientos de cada uno de los empleados, ya que estos aplicados efectivamente darán como resultados costos reducidos, un mejor servicio y productos para los clientes, obteniendo una clara ventaja competitiva en el mercado. Al tener empleados con conocimiento ayuda a tener ideas innovadoras que mejoran el desempeño organizacional en conjunto (Mahapa, 2013).

Así mismo, la organización de aprendizaje turístico ayuda a modificar y a generar un impacto positivo en las actitudes, procesos y procedimientos de los empleados, que fungen como herramienta para entregar el mejor servicio posible a sus clientes. Este tipo de servicio turístico debe ser excepcional y elementos como el uso tecnológico, el personal capacitado, la fluidez en idiomas, realización de actividades complementarias dentro del espacio, innovación organizacional y nuevo marketing turístico, ayudaran a ganar calidad y por consecuencia convertirse en una OTI, que a su vez, dependerá de la capacidad de los empleados y la aplicación de sus conocimientos al momento de satisfacer las necesidades de los clientes (Hallin y Marnburg, 2008; Ghorbani, Danaei, Mohammad Zargar y Hematian 2019).

Todos estos cambios generan capacidades nuevas, mejoradas y un conocimiento superior. Además, tiene la tendencia a convertirse en la mejor y distinguirse de otras organizaciones de turismo. Por lo que, las empresas turísticas interesadas en mejorar su ventaja competitiva, eficiencia e innovación, necesitan dirigirse a organizaciones de aprendizaje (Kraleva, 2011), donde, los avances tecnológicos, el talento humano, el capital intelectual y el giro de los servicios turísticos hacen una perfecta combinación, para lograr que las empresas turísticas adquieran el valor agregado de "inteligentes" (Ortega Herrera y Sánchez Ramos, 2018).

Por otro lado, los artículos analizados (Bayraktaroglu y Ozen Kutanis, 2003; Hallin y Marnburg, 2008; Popescu et al., 2011; Mahapa, 2013; González Macías et al., 2019) evidencian 
Organizaciones inteligentes y su incipiente incursión en la esfera turística. Una aproximación al estado del conocimiento

que las principales limitantes para la transformación de organizaciones turísticas del sector hotelero a unas de tipo inteligente son:

- Desconocimiento de su capital intelectual

- Escasez de gestión del conocimiento de forma individual como organizacional

- Ausencia de liderazgo y habilidades gerenciales para la gestión del conocimiento y aprendizaje

- Falta de previsión en el futuro para anticiparse en el desarrollo de estrategias

- Resistencia al cambio

- Alta rotación de personal

- Falta de transferencia de conocimiento entre empleados y directivos

- Clima organizacional es deficiente, lo cual limita el aprendizaje

- Falta de información interna y externa para desarrollar ventajas competitivas

Siendo que las empresas sólo se limitan a la gestión del conocimiento evidenciando una ausencia de un entorno de aprendizaje adecuado (Bayraktaroglu y Ozen Kutanis, 2003). En concreto, las problemáticas que presentan las empresas del sector turístico en su camino de transformación a inteligentes, es la prevalencia de modelos de administración tradicionales caracterizados por la unicidad de los recursos materiales y humanos limitando la transferencia del conocimiento, capacidades de absorción, apertura a los nuevos cambios, así como el aprendizaje individual y organizacional.

Sin embargo, ante escenarios adversos como el del Covid 19, las empresas turísticas fueron las primeras en sufrir las afectaciones tras la caída del turismo y como consecuencia el cierre de éstas, dejando pérdidas empresariales y una gran incertidumbre de su sobrevivencia abriendo nuevos caminos de aprendizaje ante una visión que se torna insegura y volátil. Siendo que, esta oportunidad de cambio puede llevar a las empresas turísticas a darle un enfoque de OTI, para reivindicar su concepto empresarial, su desarrollo organizacional y su conocimiento ayudando a las empresas a tomar decisiones eficaces ante las adversidades ya sean económicas, ambientales y como es el caso de la actualidad, de salud.

\section{Metodología}

Para realizar este estudio se analizó el contenido de artículos de diferentes revistas científicas como lo muestra la tabla 3 , que abordan desde conceptos generales sobre las organizaciones inteligentes, sus orígenes y sus estructuras para comprender cómo se lleva a cabo la transformación de una empresa tradicional a una inteligente, y por consecuencia que se debe considerar para evolucionar una empresa turística a una empresa turística inteligente, la cual se encuentra en un sector totalmente dinámico y versátil.

Tabla 3. Número de revistas utilizadas en la investigación

\begin{tabular}{|c|l|c|l|}
\hline $\begin{array}{c}\text { Número } \\
\text { de los } \\
\text { artículos }\end{array}$ & \multicolumn{1}{|c|}{ Nombre de la revista } & $\begin{array}{c}\text { Número de los } \\
\text { artículos }\end{array}$ & Nombre de la revista \\
\hline 1 & AD-minister & 1 & $\begin{array}{l}\text { National Institute of } \\
\text { Development Administration, } \\
\text { Thailand }\end{array}$ \\
\hline
\end{tabular}




\begin{tabular}{|c|c|c|c|}
\hline 1 & $\begin{array}{l}\text { Bibliotecas Anales de } \\
\text { Investigación }\end{array}$ & 1 & NOVATICA \\
\hline 1 & Boletín Virtual & 1 & $\begin{array}{l}\text { Participation \& Empowerment: } \\
\text { An International Journal }\end{array}$ \\
\hline 1 & $\begin{array}{l}\text { Centro de Investigación de } \\
\text { Ciencias Administrativas y } \\
\text { Gerenciales (CICAG) }\end{array}$ & 1 & Persona \\
\hline 1 & Economía industrial & 1 & $\begin{array}{l}\text { Procedia Economics and } \\
\text { Finance }\end{array}$ \\
\hline 1 & $\begin{array}{l}\text { European Journal of Training } \\
\text { and Development }\end{array}$ & 3 & $\begin{array}{l}\text { Procedia Social and Behavioral } \\
\text { Sciences }\end{array}$ \\
\hline 1 & Fidelitas & 1 & Public Administration Research \\
\hline 1 & Gestión Social & 1 & Punto de vista \\
\hline 1 & Heliyon & 1 & Quipukamayoc \\
\hline 2 & $\begin{array}{l}\text { Industrial and Commercial } \\
\text { Training }\end{array}$ & 1 & $\begin{array}{l}\text { Research-Technology } \\
\text { Management }\end{array}$ \\
\hline 1 & Informes Psicológicos & 1 & $\begin{array}{l}\text { Revista Científica Digital del } \\
\text { Centro de Investigación y } \\
\text { Estudios Gerenciales }\end{array}$ \\
\hline 1 & $\begin{array}{l}\text { International Journal of } \\
\text { Organizational Analysis }\end{array}$ & 1 & Revista de Ciencias Sociales \\
\hline 1 & $\begin{array}{l}\text { Investigaciones Europeas de } \\
\text { Dirección y Economía de la } \\
\text { Empresa }\end{array}$ & 1 & $\begin{array}{l}\text { Revista Internacional de } \\
\text { Organizaciones }\end{array}$ \\
\hline 1 & $\begin{array}{l}\text { Journal of Knowledge } \\
\text { Management }\end{array}$ & 1 & $\begin{array}{l}\text { Revista Latino América de } \\
\text { Turismologia / RELAT }\end{array}$ \\
\hline 1 & $\begin{array}{l}\text { Journal of Manufacturing } \\
\text { Technology Management }\end{array}$ & 1 & Suma de Negocios \\
\hline 1 & Journal of Marketing & 1 & Telos \\
\hline 1 & KOINONIA & 5 & The Learning Organization \\
\hline 1 & $\begin{array}{l}\text { Leadership \& Organization } \\
\text { Development Journal }\end{array}$ & 2 & Tourism Management \\
\hline 1 & Management & 1 & Turismo y Patrimonio \\
\hline 1 & Management \& marketing & 1 & Universidad \& Empresa \\
\hline 1 & Managerial Auditing Journal & 1 & UTMS Journal of Economics \\
\hline
\end{tabular}

Fuente: Elaboración propia

La revisión de las investigaciones se llevó a cabo por medio de la búsqueda de palabras clave: (en español) empresas turísticas inteligentes, organizaciones inteligentes y la gestión del conocimiento, organizaciones de aprendizaje en hoteles, organizaciones que aprenden en las empresas del sector turístico, empresas inteligentes y tecnología; (en inglés) smart organization in tourist companies, intelligent organizations, learning organizations in tourist companies, organizational structures and learning organization, smart organization in hoteles, smart organization in restaurants, smart organization in travel agency. La búsqueda se realizó a través del software de aplicación de Google Chrome que permitió localizar bases de datos que cuentan con contenidos confiables debido a sus procesos de revisión y evaluación, lo que incrementa su calidad y su impacto a nivel internacional, de las cuales se encontraron un total 
Organizaciones inteligentes y su incipiente incursión en la esfera turística. Una aproximación al estado del conocimiento

de 5,138 artículos desglosados en (tabla 4): ScienceDirect, Dialnet y Scielo; motores de búsqueda como Mendeley y Google Scholar; y finalmente con editoriales mundiales como Emerald Insight. Sin embargo, del total de los artículos hallados se realizó una exclusión de investigaciones relacionadas con organizaciones educativas inteligentes, ciudades inteligentes o mejor conocidas como Smart City, hospitales inteligentes, así como, destinos inteligentes en turismo, dejando un total de 50 artículos para analizar, de ellos fueron 29 investigaciones de un periodo de diez años (2010-2020) que permitieron identificar cómo el concepto de organización inteligente está inserto en la actualidad, en donde las empresas tienen constantes cambios y las necesidades son latentes, y 21 investigaciones con un rango de años entre 1996-2009, que abarcan temas sobre el origen y la evolución de este concepto, los cuales se empezaron a desarrollar en las empresas identificando a su vez la importancia de convertirse en una organización inteligente.

Tabla 4. Artículos encontrados y excluidos por bases de datos

\begin{tabular}{|l|c|c|c|}
\hline \multicolumn{1}{|c|}{ Base de datos } & $\begin{array}{c}\text { Artículos } \\
\text { encontrados }\end{array}$ & Artículos excluidos & $\begin{array}{c}\text { Artículos por } \\
\text { analizar }\end{array}$ \\
\hline ScienceDirect & 2,971 & 2,963 & 8 \\
\hline Dianelt & 500 & 491 & 9 \\
\hline Scielo & 250 & 248 & 2 \\
\hline Mendeley & 531 & 524 & 7 \\
\hline Google Scholar & 660 & 650 & 10 \\
\hline Emerald Insigh & 226 & 212 & 14 \\
\hline
\end{tabular}

Fuente: elaboración propia.

Por consiguiente, se analizaron y clasificaron los artículos según su perfil temático, los cuales fueron agrupados en cuatro principales categorías:

1. Organizaciones tradicionales

2. Organizaciones que aprenden u organizaciones inteligentes

3. Análisis organizacional

4. Organizaciones turísticas inteligentes

Así también, a través del software VOSviewer, se realizó un estudio bibliometrico que de acuerdo a autores como Benckendorff y Zehrer (2013); Koseoglu, Rahimi, Okumus y Jingyan, (2016); Naveen, Satish, Debmalya, Nitesh y Weng (2021), es un análisis cuantitativo que consiste en mapear el conocimiento por medio de los componentes de varias investigaciones, por ejemplo, las disciplinas de estudio, citas, autores, palabras clave y métodos empleados, estableciendo así, datos más estructurados para comprender las interrelaciones de los estudios y de los impactos que genera de forma intelectual. $Y$ aunque en el campo turístico estos análisis bibliometricos han tenido un aumento considerable en los últimos años es importante su aplicación, debido a que el turismo es multidisciplinario y puede aumentar los conocimientos con base académico, así como promover mejores estrategias y prácticas en el campo laboral y profesional.

Por tanto, se creó un mapa de co-ocurrencia de términos, basado en datos de textos de referencias, los campos de donde se extrajeron los términos fueron el título y los resúmenes 
de los artículos, ignorando etiquetas abstractas estructuradas y declaraciones de derechos de autor, todo bajo el método de conteo completo, con un número mínimo de co-ocurrencias de 5 de los 1,204 términos, donde 22 alcanzan el mínimo. Cada uno de los 22 términos, se calcularon con un $60 \%$ de los términos más relevantes. Tal como se muestra en la figura 1.

Figura 1. Términos más relevantes

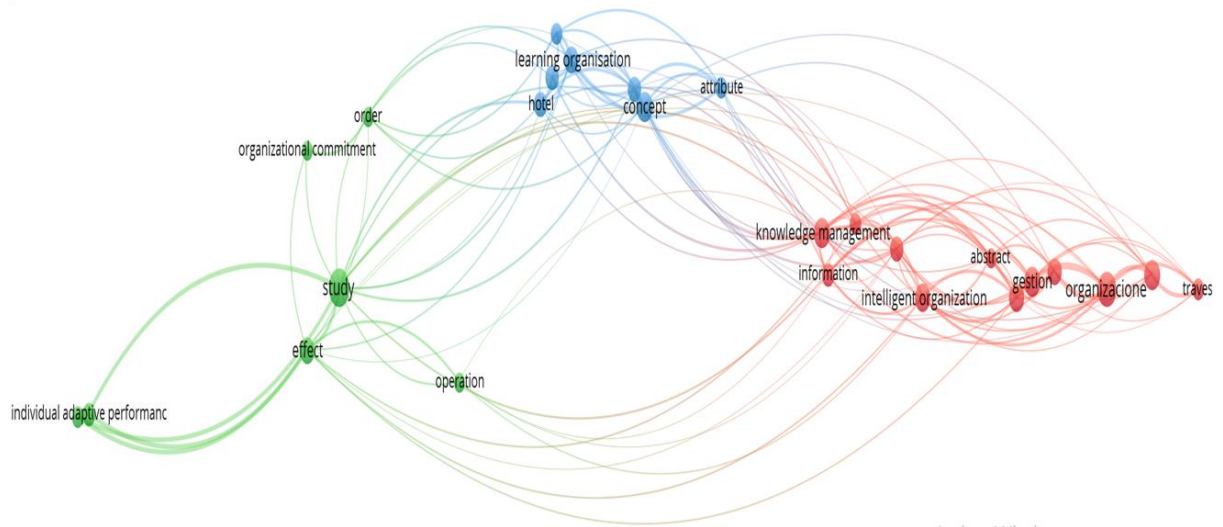

Fuente: Vosviewer.

Además, en la figura 2, se muestra la red de autores de acuerdo a la co-ocurrencia e interrelaciones que existe entre los autores en este campo de investigación específicamente entre los años de 2012-2020 donde se centran los mayores estudios.

Figura 2. Red de autores por co-ocurrencia por años

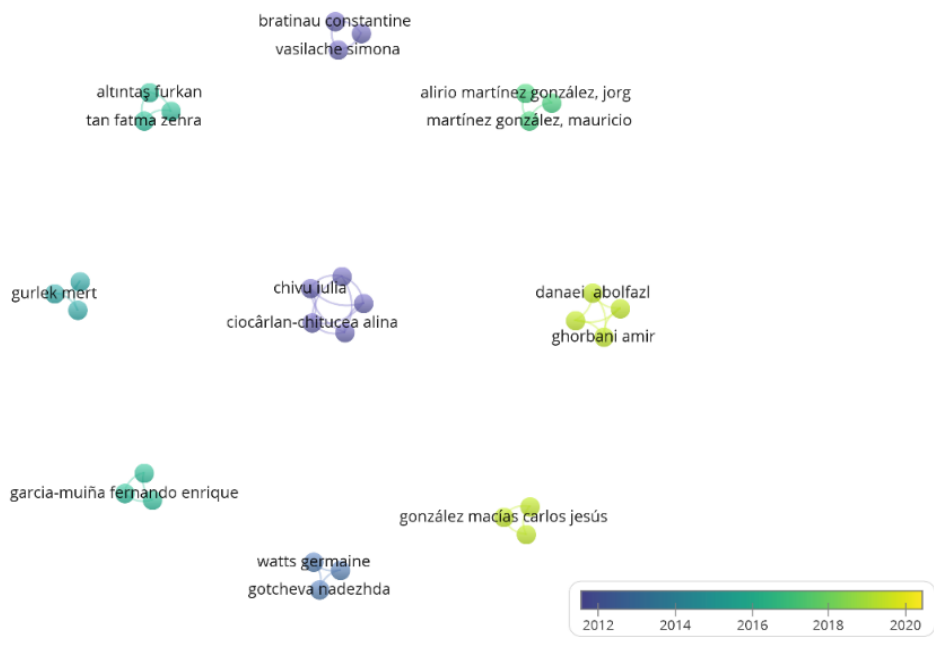

Fuente: VOSviewer 
Organizaciones inteligentes y su incipiente incursión en la esfera turística. Una aproximación al estado del conocimiento

En cuanto a la coautoria se logró identificar solamente un clúster, como lo menciona la tabla 5 .

Tabla 5. Red coautoria

\begin{tabular}{|c|}
\hline Clúster 1 \\
\hline Chivu lulia \\
\hline Ciocârlan-Chitucea Alina \\
\hline Georgel Călin \\
\hline Popescu Dan \\
\hline Popescu Daniela Oana \\
\hline
\end{tabular}

Fuente: VOSviewer

Siendo este mismo grupo de autores quienes han incursionado en el estudio de organizaciones que aprenden en el ámbito turístico.

Posteriormente, se desglosó el concepto y los principios básicos de una organización inteligente, para llevarla al contexto turístico. Al respecto de los 50 artículos encontrados, se detectaron tan sólo diez investigaciones enfocadas al sector hotelero como empresa turística, los cuales se eligieron por incluir conceptos como: organizaciones de aprendizaje, gestión del conocimiento, organización inteligente y organización turística inteligente. Resaltando que este concepto es una gestión contemporánea que define cómo debe operar una organización en un negocio en constante cambio como es el turismo, tabla 6.

Tabla 6. Principales estudios de Empresas Inteligentes Turísticas (2003-2019)

\begin{tabular}{|l|l|c|}
\hline \multicolumn{1}{|c|}{ Título de la investigación } & \multicolumn{1}{|c|}{ Autor (es) } & $\begin{array}{c}\text { Año de } \\
\text { investigación }\end{array}$ \\
\hline $\begin{array}{l}\text { Transformar los hoteles en organizaciones } \\
\text { de aprendizaje: una nueva estrategia para } \\
\text { globalizarse. }\end{array}$ & $\begin{array}{l}\text { Bayraktaroglu y Ozen Kutanis } \\
(2003)\end{array}$ & 2003 \\
\hline $\begin{array}{l}\text { Cultura de aprendizaje individual y de } \\
\text { organización en organizaciones de } \\
\text { aprendizaje hoteles de cinco estrellas en la } \\
\text { región de Antalya (Turquía). }\end{array}$ & Aksu y Özdemir (2005) & 2005 \\
\hline $\begin{array}{l}\text { Gestión del conocimiento en la industria de } \\
\text { la hostelería: Una revisión de la } \\
\text { investigación empirica. }\end{array}$ & Hallin y Marnburg (2008) & 2008 \\
\hline $\begin{array}{l}\text { Organizaciones de aprendizaje: Requisito } \\
\text { previo para organizaciones de turismo } \\
\text { exitosas. }\end{array}$ & Kraleva (2011) & 2011 \\
\hline $\begin{array}{l}\text { Los desafíos de la organización de } \\
\text { aprendizaje en las PYMES campo de } \\
\text { actividad del turismo. }\end{array}$ & $\begin{array}{l}\text { Popescu, Chivu, Ciocârlan- } \\
\text { Chitucea, Popescu y Georgel } \\
\text { (2011) }\end{array}$ & 2011 \\
\hline
\end{tabular}




\begin{tabular}{|l|l|c|}
\hline $\begin{array}{l}\text { Impacto de las estrategias de gestión del } \\
\text { conocimiento en el desempeño } \\
\text { organizacional en la industria hotelera de } \\
\text { Zimbabwe }\end{array}$ & 2013 \\
\hline $\begin{array}{l}\text { Efecto de los factores de intercambio de } \\
\text { conocimientos sobre la organización del } \\
\text { aprendizaje en turismo y hotelería }\end{array}$ & Suveatwatanakul (2013) & 2013 \\
\hline $\begin{array}{l}\text { La organización inteligente como apuesta } \\
\text { de evolución en la industria turística de } \\
\text { México. }\end{array}$ & $\begin{array}{l}\text { Ortega Herrera y Sánchez Ramos } \\
(2018)\end{array}$ & 2018 \\
\hline $\begin{array}{l}\text { Diseño de una organización de turismo } \\
\text { inteligente (STO) para la gestión del turismo: } \\
\text { un estudio de caso de organizaciones de } \\
\text { turismo de la provincia de Khorasan del Sur, } \\
\text { Irán. }\end{array}$ & \begin{tabular}{l} 
Ghorbani, Danaei, Mohammad y Hematian (2019) \\
\hline $\begin{array}{l}\text { Gestión del conocimiento enfocado a la } \\
\text { competitividad en empresas familiares } \\
\text { turísticas (EFT) de Guanajuato, México. }\end{array}$
\end{tabular} & $\begin{array}{l}\text { González Macías, Guerrero } \\
\text { Rodríguez y Vidaurri Aréchiga, } \\
(2019)\end{array}$ \\
\hline
\end{tabular}

Fuente: Elaboración propia.

En conjunto estos artículos recalcan que para convertirse en organizaciones turísticas inteligentes es necesario dos elementos importantes, el primero de ellos, es la gestión del conocimiento, el cual no se tiene que ver como un gasto, sino como una inversión, porque interviene en la eficiencia y en la competitividad de la empresa, dando como resultado un trabajo en equipo donde se desarrollan programas, actividades de comunicación, capacitaciones, políticas, procedimientos, así como la intervención del uso tecnológico, que generen un flujo de la información constante, agregando de tal forma un valor integrado que aumenta la calidad en los productos o servicios, derivado a la interacción precisa entre prestador de servicio y cliente, para así satisfacer las necesidades de los consumidores. El segundo es el liderazgo, como aquel que impulsa el rendimiento y el compromiso del personal, dirigiéndolos hacia los objetivos y metas de la empresa, acompañado de normas a seguir para que la calidad de lo que ofrece sea uno de los mejores estándares de su ventaja competitiva.

De igual manera se logra evidenciar que el interés por el estudio de las Organizaciones Turísticas Inteligentes (OTI) ha crecido después de que diversos autores han concretado el concepto de una organización inteligente, y que ésta se puede llevar a diversos sectores donde el turismo no es la excepción, ya que en este sentido se busca que la empresa se transforme internamente para brindarle al cliente un valor en su producto o servicio alcanzando sus expectativas, y así se vuelva más competitiva en el mercado. Aunque cabe señalar que los estudios siguen siendo escasos, debido a que se limitan al ámbito hotelero, cuando el turismo abarca desde sectores restauranteros, hoteleros, agencias de viajes, cruceros, aerolíneas, solo por mencionar algunos, y en donde también existe la posibilidad de convertirse en una organización turística inteligente.

\section{Conclusiones y futuras líneas de investigación}

Las empresas inteligentes como un campo de estudio, ha sido objeto de investigación global, siendo reciente la participación de los países de Centroamérica y Latinoamérica, mientras 
Organizaciones inteligentes y su incipiente incursión en la esfera turística. Una aproximación al estado del conocimiento

que los estudios base para la construcción del concepto son en idioma inglés y de países de Norteamérica y Europa. También, en el caso de las empresas turísticas, los hallazgos son limitados. Después de la revisión de la literatura, principalmente se encuentran conceptos teóricos, mientras que la evidencia empírica aún es limitada más en el ámbito turístico y se usan como sinónimos palabras como organizaciones que aprenden y organizaciones inteligentes.

Tanto en Europa como en Estados Unidos, han surgido escuelas que buscan explicar el concepto a partir de sus experiencias empresariales, no sólo de forma individual sino por región. La generalidad de los estudios profundiza sobre conceptos de aprendizaje organizacional, liderazgo y gestión del conocimiento principalmente, concluyendo que, los efectos de la inteligencia en las organizaciones se traducen generalmente en mayor competitividad, flujo de información y productividad dentro de la empresa.

Existe otra ola de estudios que hace énfasis en la necesidad de transformar empresas catalogadas como tradicionales a inteligentes donde el aprendizaje y la tecnología se integran como una herramienta para manejar la información, vinculándola al manejo tanto de las estrategias como de la innovación organizacional, basándose en las características internas como externas de la empresa que logra cubrir las necesidades empresariales y del mercado. Desde esta óptica, las empresas del sector turístico se han caracterizado por tener como prioridad su estabilidad en el mercado mediante estrategias de comercialización que le ayuden a satisfacer las necesidades de los clientes de acuerdo a las tendencias actuales para obtener ventajas competitivas, ofreciendo a su vez reducción de costos empresariales y siendo responsable con el medio ambiente, el cual es un atractivo para los clientes.

Los estudios sobre empresas inteligentes tienen aplicación notoria en el ramo turístico, industria que enfrenta grandes retos, complejos escenarios y cambios en los hábitos de consumo del turista. Donde el rápido aprendizaje individual como organizacional a través de los activos intelectuales y la reconceptualización de la prestación del servicio de forma innovadora, definirán qué empresas pueden evolucionar, aprender, adaptarse, desarrollar habilidades y ser inteligentes, incluso a la capacidad de anticiparse y crear nuevas necesidades en los consumidores, dando como resultado un atractivo en el producto o servicio, debido a que la empresa genera únicas e inimitables ventajas competitivas.

La multifactoriedad del concepto Ol, así como la integración del recurso humano, el aprendizaje organizacional, el liderazgo, el clima y la cultura organizacional hace que no se identifiquen modelos de negocios claros para la transformación de empresas tradicionales a inteligentes. Más aún, dependiendo del autor se hace énfasis en el aprendizaje, dirección o liderazgo, sin que se pueda identificar cual cobra mayor peso en el proceso de reconversión empresarial.

Con una visión diferente centrada en la innovación se aborda la capacidad de aprendizaje e intercambio de conocimiento como medio para estar mejor preparados para responder a las presiones externas, y mejorar la imagen corporativa al orientarse más a la gente. Sin embargo, en los estudios no se vincula directamente la innovación como generadora de empresas inteligentes. Además de las características anteriores, se deben complementar con tecnología, gestión de la información, auto transformación, experimentación, proyección, transdisciplinariedad, disminución jerárquica, visión de la empresa, que garanticen el éxito de la empresa (Becerra y Sánchez, 2011) 
Así también son recurrentes las posturas de que la transformación empresarial estará gestionada por el departamento de recursos humanos, buscando proporcionar intervenciones eficaces para mejorar el comportamiento innovador tras la implementación de cambios organizativos, dedicando esfuerzos para crear un lugar de trabajo el cual promueva la cultura eficaz, de aprendizaje colaborativo, así como un compromiso laboral en actividades y programas relevantes de la empresa en donde el personal desarrolle habilidades y competencias individuales, que los lleve incluso a la participación de toma de decisiones dando como resultado el éxito de la empresa (Claver Cortés y Zaragoza Sáez, 2007; Kyoung Park, Hoon Song, Won Yoon y Kim, 2014). Por tanto, el futuro de las organizaciones está a cargo de las personas, quienes poseen el conocimiento, destrezas y habilidades que generan un valor económico incalculable para las organizaciones, dándole un dominio de la información para que las empresas obtengan una gran ventaja competitiva frente a sus competidores, aportando nuevos productos o servicios, acortando los procesos de producción con el menor tiempo de entrega y costo con la mayor cantidad de ingresos posibles, lo cual no sería posible sin ese activo intangible tan importante e indispensable para el desarrollo y permanencia en el mercado confirmando lo planteado por González Beuses (2019).

\section{Referencias bibliográficas}

Adamczewski, Piotr (2016). ICT solutions in intelligent organizations as challenges in a knowledge economy. Revista Management. Volumen 20, No. 2. Polonia (Pp. 198209). https://doi.org/10.1515/manment-2015-0060

Aksu, A. Akin; Özdemir, Bahattin (2005). Individual learning and organization culture in learning organizations: Five star hotels in Antalya region of Turkey. Revista Managerial Auditing Journal. Volumen 20, No. 4. Turquia (Pp. 422-441). https://doi.org/10.1108/02686900510592098

Amy, H. Amy (2008). Leaders as facilitators of individual and organizational learning. Revista Leadership \& Organization Development Journal. Volumen 29, No. 3. Estados Unidos (Pp. 212-234). https://doi.org/10.1108/01437730810861281

Angulo Rincón, Rosalba (2017). Gestión del conocimiento y aprendizaje organizacional: una visión integral. Revista Informes Psicológicos. Volumen 17, No. 1. Colombia (Pp. 53-70). http://dx.doi.org/10.18566/infpsic.v17n1a03

Atak, Metin; Erturgut, Ramazan (2010). An empirical analysis on the relation between learning organization and organizational commitment. Revista Procedia Social and Behavioral Sciences. No. 2. Turquía (Pp. 3472-3476). https://doi.org/10.1016/i.sbspro.2010.03.537

Bahar Aşcl, Hatice; Zehra Tan, Fatma; Altıntaş, Furkan (2016). A Strategic Approach For Learning Organizations: Mental Models. Revista Procedia Social and Behavioral Sciences. No. 235. Turquia (Pp. 2 -11). https://doi.org/10.1016/j.sbspro.2016.11.002 Bayraktaroglu, Serkan; Ozen Kutanis, Rana (2003). Transforming hotels into learning organisations: a new strategy for going global. Revista Tourism Management. No. 24. Turquía (Pp. 149-154). https://doi.org/10.1016/S0261-5177(02)00061-4

Becerra, Marlene; Sánchez, Ludy (2011). El liderazgo en las organizaciones inteligentes. Revista Científica Digital del Centro de Investigación y Estudios Gerenciales. Volumen 1, No. 4. Venezuela (Pp. 61-71). 
Organizaciones inteligentes y su incipiente incursión en la esfera turística. Una aproximación al estado del conocimiento

https://www.grupocieg.org/archivos_revista/1-4\%20(61-

71)\%20becerra\%20sanchez $\% 20$ rcieg\%20mayo\%2011_articulo_id29.pdf

Benckendorff, Pierre; Zehrer, Anita (2013). A network analysis of tourism research. Revista Annals of Tourism Research. Volumen 43. Reino Unido (Pp. 121-149). https://doi.org/10.1016/j.annals.2013.04.005

Bhatt, Ganesh D. (2001). Knowledge management in organizations: examining the interaction between technologies, techniques, and people. Revista Journal of Knowledge Management. Volumen 5, No. 1. Estados Unidos (Pp. 68-75). https://doi.org/10.1108/13673270110384419

Brătianu, Constantin; Vasilache, Simona; Jianu, lonela (2006). In search of intelligent organizations. Revista Management \& marketing. No. 4. Bucarest (Pp. 71-82). https://www.researchgate.net/publication/227430437_In_search_of intelligent_organi zations

Chacón León, Luz Ma. (2017). La organización por procesos en empresas de servicios: un abordaje teórico. Revista Fidelitas. No. 7. Costa Rica (Pp. 173-195). https://revistas.ufidelitas.ac.cr/index.php/revista_fidelitas/article/view/13

Chávez Hernández, Noé; Torres Sanabria, Guillermo (2012). La organización inteligente en un ambiente de aprendizaje: una exploración de sus aspectos generales. Revista ADminister. No. 21. Colombia (Pp. 101-115). http://www.scielo.org.co/scielo.php?script=sci_arttext\&pid=S169202792012000200007

Claver Cortés, E; Zaragoza Sáez, P. (2007). La dirección de recursos humanos en organizaciones inteligentes. Una evidencia empírica desde la dirección del conocimiento. Revista Investigaciones Europeas de Dirección y Economía de la Empresa. Volumen 13, No. 2. España (Pp. 55-73). https://dialnet.unirioja.es/servlet/articulo?codigo $=2356653$

Gallego, Domingo J; Gil, Alfonso J. (2012). La construcción de organizaciones de aprendizaje a través del liderazgo. Revista Universidad \& Empresa. No. 22. Colombia (Pp. 43-77). https://revistas.urosario.edu.co/index.php/empresa/article/view/1892

Gardiner, Penny; Whiting, Peter (1997). Success factors in learning organizations: an empirical study. Revista Industrial and Commercial Training. Volumen 29, No. 2. Reino Unido (Pp. 41-48). http://dx.doi.org/10.1108/00197859710165001

Ghorbani, Amir; Danaei, Abolfazl; Mohammad Zargar, Seyed; Hematian, Hadi (2019). Designing of smart tourism organization (STO) for tourism management: A case study of tourism organizations of South Khorasan province, Iran. Revista Heliyon. Volumen 5, No. 6. Irán (Pp. 1-9). https://doi.org/10.1016/j.heliyon.2019.e01850

Gil López, Alfonso J; Ruiz, Vicente Lázaro (2016). Influencia de los principios de aprendizaje organizativo sobre la eficacia de las organizaciones que Aprenden. Revista Economía industrial. $\quad$ No. $400 . \quad$ España (Pp. 161-171). https://www.mincotur.gob.es/Publicaciones/Publicacionesperiodicas/Economialndustr ial/RevistaEconomialndustrial/400/ALFONSO\%20GIL\%20y\%20V.\%20LAZARO.pdf 
Gillezeau, Patricia (1999). La cultura organizacional en el desarrollo de empresas inteligentes. Fundamentos: valores, comunicación y liderazgo. Revista Telos. Volumen 1, No. 2. Venezuela (Pp. 221-232). http://ojs.urbe.edu/index.php/telos/article/view/934

González Beuses, Annayeskha Grabiela (2019). Incidencia de las Organizaciones Inteligentes en la Gestión del Conocimiento. Revista Arbitrada Interdisciplinaria KOINONIA. Volumen 4, No. 8. Venezuela (Pp. 366-392). http://dx.doi.org/10.35381/r.k.v4i8.283

González Macías, Carlos Jesús; Guerrero Rodríguez, Rafael; Vidaurri Aréchiga, José Eduardo (2019). Gestión del conocimiento enfocado a la competitividad en empresas familiares turísticas (EFT) de Guanajuato, México. Revista Turismo y Patrimonio. No. 13. México

http://ojs.revistaturismoypatrimonio.com/index.php/typ/article/view/189

13-34).

Gotcheva, Nadezhda; Watts, Germaine; Oedewald, Pia (2013). Developing smart and safe organizations: an evolutionary approach. Revista International Journal of Organizational Analysis. Volumen 21, No. 1. Europa (Pp. 83-97). https://doi.org/10.1108/19348831311322551

Hallin, Carina Antonia; Marnburg, Einar (2008). Knowledge management in the hospitality industry: A review of empirical research. Revista Tourism Management. Volumen 29. Noruega (Pp. 366-381). https://www.sciencedirect.com/science/article/abs/pii/S026151770700043X

Hill, Rosemary (1996). A measure of the learning organization. Revista Industrial and Commercial Training. Volumen 28, No. 1. Reino Unido (Pp. 19-25). http://dx.doi.org/10.1108/00197859610105440

Kanten, Pelin; Kanten, Selahattin; Gurlek, Mert (2015). The Effects of Organizational Structures and Learning Organization on Job Embeddedness and Individual Adaptive Performance. Revista Procedia Economics and Finance. Volumen 23. Turquía (Pp. 1358-1366). https://www.sciencedirect.com/science/article/pii/S2212567115005237

Khan, Urfi; Haleem, Abid (2015). Improving to smart organization: an integrated ism and fuzzymicmac modelling of barriers. Revista Journal of Manufacturing Technology Managment. Volumen 26, No. 6. India (807-829). http://dx.doi.org/10.1108/JMTM-052014-0062

Koseoglu, Mehmet Ali; Rahimi, Roya; Okumus, Fevzi; Jingyan, Liu (2016). Bibliometric studies in tourism. Revista Annals of Tourism Research. Volumen 61. Estados Unidos (Pp. 180-198). https://doi.org/10.1016/j.annals.2016.10.006

Kraleva, Natasa (2011). Learning organizations: Prerequisite for successful tourism organizations. Revista UTMS Journal of Economics. Volumen 2, No. 1. Macedonia (Pp. 77-82). https://www.econstor.eu/handle/10419/49233

Kyoung Park, Yu; Hoon Song, Ji; Won Yoon, Seung; Kim, Jungw0o (2014). Learning organization and innovative behavior. The mediating effect of work engagement. Revista European Journal of Training and Development. Volumen 38, No. 1/2. Estados Unidos (Pp. $75-$ 94). https://doi.org/10.1108/EJTD-04-2013-0040

Larrota Castro, Sandra Yamile (2012). La evolución del conocimiento en las organizaciones inteligentes. Revista Punto de vista. Volumen 3, No. 5. Colombia (Pp. 119 - 138). https://doi.org/10.15765/pdv.v3i5.136 
Organizaciones inteligentes y su incipiente incursión en la esfera turística. Una aproximación al estado del conocimiento

López-Zapata, Esteban; García-Muiña, Fernando Enrique; García-Moreno, Susana María (2016). Atributos de la organización que aprende: una revisión de la literatura. Revista Internacional de Organizaciones. No. 16. España (Pp. 59-81). https://doi.org/10.17345/rio16.59-81

Mahapa, Mildred (2013). Impact of Knowledge Management Strategies on Organizational Performance in the Hospitality Industry of Zimbabwe. Revista Public Administration Research. Volumen 2, No. 1. Zimbabwe (Pp. 76-83). https://www.ccsenet.org/journal/index.php/par/article/view/26821

Martínez González, Mauricio; Gómez Pérez, Henry; Martínez González, Jorge Alirio (2017). La gestión de la incertidumbre: Empresas inteligentes con trabajadores del conocimiento. Revista Boletín Virtual. Volumen 6, No. 8. Colombia (Pp. 132-143). https://dialnet.unirioja.es/servlet/articulo?codigo $=6224319$

Matheson, David; Matheson, James E. (2001). Smart Organizations Perform Better. Revista Research-Technology Management. Volumen 44, No. 4. Estados Unidos (Pp. 4954). http://dx.doi.org/10.1080/08956308.2001.11671442

Mendoza Pineda, Myrian Teresa (2011). La gestión del conocimiento y el capital intelectual como base fundamental de las organizaciones inteligentes. Revista Gestión y Sociedad. Volumen 4, No. 1. Colombia (Pp. 17-25). https://xdoc.mx/documents/la-gestion-delconocimiento-y-el-capital-intelectual-como-5e35de6e8a260

Moh'd, Raid; Dahou, Khadra; Hacini, Ishaq (2016). The impact of knowledge conversion processes on implementing a learning organization strategy. Revista The Learning Organization. Volumen 19, No. 6. Argelia (Pp. 482-496). https://doi.org/10.1108/09696471211266947

Naveen, Donthu; Satish, Kumar; Debmalya, Mukherjee; Nitesh, Pandey; Weng, Marc Lim (2021). How to conduct a bibliometric analysis: An overview and guidelines. Revista Journal of Business Research. Volumen 133. Estados Unidos (Pp. 285-296). https://doi.org/10.1016/j.jbusres.2021.04.070

Ortega Herrera, Alejandro Misael; Sánchez Ramos, María Eugenia (2018). La Organización Inteligente como apuesta de evolución en la industria Turística De México. Revista Latino América de Turismologia / RELAT. Volumen 4, No. 2. México (Pp. 73-82). https://doi.org/10.34019/2448-198X.2018.v4.14037

Pérez-Soltero, Alonso (2006). El papel de las Tecnologías de Información y la memoria organizacional dentro de las Empresas Inteligentes. Revista NOVATICA. No. 182. México (Pp. 52-55). http://www.aperez.mx/novatica182_2006.pdf

Popescu, Dan; Chivu, Iulia; Ciocârlan-Chitucea, Alina; Popescu, Daniela-Oana; Georgel, Călin (2011). The learning organization challenges within the SMEs tourism field of activity.

Revista Procedia Social and Behavioral Sciences. No. 24. Rumania (Pp. 10981106). https://doi.org/10.1016/..sbspro.2011.09.042

Rodríguez Cruz, Yunier (2016). Concepción estratégica de la Gestión de Información y del Conocimiento para organizaciones inteligentes. Revista Bibliotecas Anales de Investigación. Volumen 12, No. 2. Cuba (Pp.165-181). https://dialnet.unirioja.es/servlet/articulo?codigo $=5766703$ 
Rojas, Jenifer (2018). Capital Humano: Un desglose teórico para su operatividad en Organizaciones Inteligentes. Centro de Investigación de Ciencias Administrativas y Gerenciales (CICAG). Volumen 16, No. 1. Venezuela (Pp. 43-54). https://dialnet.unirioja.es/servlet/articulo?codigo $=6844337$

Serrano Orellana, Bill Jonathan; Portalanza, Alexandra (2014). Influencia del liderazgo sobre el clima organizacional. Revista Suma de Negocios. Volumen 5, No. 11. España (Pp. 117-125). https://www.elsevier.es/es-revista-suma-negocios-208-articulo-influenciadel-liderazgo-sobre-el-S2215910X14700266

Slater, Stanley F; Narver, John C. (1995). Market Orientation and the Learning Organization. Revista Journal of Marketing. Volumen 59. Estados Unidos (Pp. 63-74). https://doi.org/10.2307/1252120

Solf Zárate, Arturo (2007). La organización que aprende y su aporte al proceso de cambio. Revista Persona. No. $10 . \quad$ Perú (Pp. 29-47). https://www.redalyc.org/articulo.oa?id=147112813003

Stewart, Deb (2001). Reinterpreting the learning organisation. Revista The Learning Organization. Volumen 8, No. 4. Australia (Pp. 141-152). http://dx.doi.org/10.1108/EUM0000000005607

Stonehouse, George H; Pemberton, Jonathan D. (1999). Learning and knowledge management in the intelligent organisation. Revista Participation \& Empowerment: An International Journal. Volumen 7, No. 5. Reino Unido (Pp. 131-144). http://dx.doi.org/10.1108/14634449910287846

Su-Chao, Chang y Ming-Shing, Lee (2007). A study on relationship among leadership, organizational culture, the operation of learning organization and employees' job satisfaction. Revista The Learning Organization. Volumen 14, No. 2. Taiwan (Pp. 155-185). https://doi.org/10.1108/09696470710727014

Suveatwatanakul, Chokechai (2013). Effect of knowledge sharing factors on the learning organisation in tourism and hospitality. National Institute of Development Administration, Thailand. Tailandia (Pp. 718-730). https://www.semanticscholar.org/paper/EFFECT-OF-KNOWLEDGE-SHARINGFACTORS-ON-THE-LEARNINGSuveatwatanakul/40f4e2f7f9b2f7f6d9c58a83f28f6036a1990149

Talla Ramos, Sabino (1998). El proceso de Cambio organizativo y Las organizaciones inteligentes. Revista Quipukamayoc. Volumen 5, No. 10. Perú (Pp. 51-66). DOI: https://doi.org/10.15381/quipu.v5i10.5950

Thomas, Keith; Allen, Stephen (2006). The learning organisation: a meta-analysis of themes in literature. Revista The Learning Organization. Volumen 13, No. 2. Australia (Pp. 123139). https://doi.org/10.1108/09696470610645467

Valecillos, César A; Quintero, Niria (2007). Enfoque de las organizaciones inteligentes en la implementación de nuevas técnicas de dirección en las pequeñas y medianas empresas (PYMEs). Revista de Ciencias Sociales. Volumen 13, No. 2. Venezuela (Pp. 278-289). http://ve.scielo.org/scielo.php?script=sci_arttext\&pid=S1315$\underline{95182007000200007}$ 
Organizaciones inteligentes y su incipiente incursión en la esfera turística. Una aproximación al estado del conocimiento

Weldy, Teresa G. (2009). Learning organization and transfer: strategies for improving performance. Revista The Learning Organization. Volumen 16, No. 1. Estados Unidos (Pp. 58-68). http://dx.doi.org/10.1108/09696470910927678 